\title{
EVALUATION OF COMBINING ABILITY AND HETEROSIS IN VARIOUS TRAITS OF ZEA MAYS HYBRIDS
}

\author{
MASOOD, M. ${ }^{1 *}-$ AHSAN, M. ${ }^{1}-$ SADAQAT, H. A. ${ }^{1}-$ AwAN, F. S. ${ }^{2}$ \\ ${ }^{I}$ Department of Plant Breeding and Genetics, University of Agriculture, Faisalabad, Pakistan \\ ${ }^{2}$ Centre of Agriculture Biochemistry and Biotechnology, University of Agriculture, Faisalabad, \\ Pakistan \\ *Corresponding author \\ e-mail: mmasoodmessage@gmail.com
}

(Received 28 $8^{\text {th }}$ Nov 2019; accepted 23 ${ }^{\text {rd }}$ Mar 2020)

\begin{abstract}
The selection of parents and superior genotypes is a primary task for plant breeders. In line $\times$ tester mating design, seven lines and three testers were crossed to create twenty-one hybrids. The hybrids and parents were evaluated together in the field for various yield and yield related traits at maturity under water stress conditions. The variance assessment implies prominent contrasts between the lines, and testers, crosses for various traits. The differences for GCA and SCA were observed significant for the traits. The line WM13RA and OH33-1 showed the highest general combining ability for most of the traits. The cross combination WM13RA $\mathrm{C} \times$ Agati85 provided the highest positive better parents heterosis. Specific combining ability was also high, ML17 $\times$ Agati85 and ML3 $\times$ Agati85 produced higher grain yield in term of said production of early maturing hybrids. Concerning molecular component, ten SSR primers were used to check the hybrid purity. One primer showed the polymorphism with parents and hybrids, results showed that seventeen hybrids were confirmed based on the presence of the polymorphic bands. Genetic purity was $80.95 \%$.
\end{abstract}

Keywords: combining ability, line $\times$ tester, maize, heterosis, SSR markers, water stress

\section{Introduction}

Agriculture is a main pillar of the economy in Pakistan adding $0.85 \%$ to GDP. Maize is the third largest cereal crop in Pakistan (Anonymous, 2019). It is grown on an area of 191 million ha and 1104 million metric tons are produced with a yield of 5.77 metric tons/ha (FAO, 2019). Maize is grown on an area of about 1318 thousand ha with grain production of 6.309 million tons in Pakistan. Punjab and Khyber Pakhtunkhwa (KPK) contribute significantly to maize production in Pakistan. The Province Punjab share $56.76 \%$ growing area with $80.12 \%$ grain production while KPK share $42.52 \%$ crop growing area with $19.59 \%$ grain production (Anonymous, 2019). In Punjab it is grown in spring and autumn but only in the spring season in KPK because of no space crop pattern (Rana, 2014). Maize is also a " $5 \mathrm{~F}$ " crop, i.e. food, feed, fuel, fiber and fodder crop. Its insoluble fiber used to cure the acidity of stomach. It is backbone of the feed for the poultry. Maize grain as food contains protein, starch, fiber, oil, sugar, ash 11, 71, 5.7, 4.8, 3.0 and 1.7\%, respectively (Chaudhary, 1983). Pakistan generated about 52 thousand million liters of ethanol while the world produce 28.9 million liters (Anonymous, 2019). Maize fodder can be fed to animals at any time after sowing without toxicity of chemicals (Dahmardeh et al., 2009). The demand of maize increases as the population increase, due to multiple usage of the maize grains as food for human while maize fodder as feed for animals (Mishra and Cherkauer, 2010) Pakistan has deep rich soil in South Asia and it is one of the densely packed nations of the world. Agricultural production stayed low in 2018-19. The underperformance of the agricultural sector was due to a decrease in the cultivation region, a decrease in the supply 
of water and a decrease in fertilizer usage (Aaliya et al., 2016; Ali et al., 2015, 2017). Abiotic stresses make large area unfit for agriculture due to adverse effects such as drought. In the Punjab plain and Sindh provinces, there is low precipitation $(<200 \mathrm{~mm})$ and warm spell throughout the year (Ali et al., 2013, 2016). Due to the enormous exploitation of fresh reservoirs, water shortages are severe threat to our agriculture. Drought has a serious impact on the development of maize plant (Wynne et al., 1970). Pakistan is unable to fulfill the requirements of the maize seed. The demand of maize seed is about 28892 metric tons from which the public sector provides 237 metric tons, the private sector companies provide 1222 metric tons, while 12776 metric tons of seeds is imported, in total 14235 metric tons are available. The supply of seed is about $50 \%$ of the demand so there is a shortage of seeds (Anonymous, 2019). There are various threats for maize grain and fodder yield throughout the globe, there is a need to develop such maize genotypes and hybrids which can produce higher grain yield. The present study is designed for the selection of the parents to develop local hybrids and synthetic varieties on the basis of combining ability and heterosis and to confirm hybrid nature with molecular analysis.

\section{Materials and methods}

The seven screened out tolerant lines (which showed higher root length, shoot length and ratio of root shoot length, were selected as tolerant maize genotypes) were grown during the spring season of 2016 and hybridized with three testers to produce 21 hybrids line $\times$ tester mating design. The location and average climate during the research period were the followings; Latitude $=31^{\circ} 44^{\prime} \mathrm{N}$, Longitude $=73^{\circ} 06^{\prime} \mathrm{E}$, Altitude $=184.4 \mathrm{~m}$, the average temperature was $35.7^{\circ} \mathrm{C}$, rain fall was $2.6 \mathrm{~mm}$, pan evaporation was $8.4 \mathrm{~mm}$, sun shine was $12.4 \mathrm{~h}$, ETO was $7.9 \mathrm{~mm}$ and wind speed was $6.23 \mathrm{~km} / \mathrm{h}$. Both (parents and hybrids) were grown in triplicate randomized complete block design in the field during the autumn of 2016 and the performance of the hybrids were evaluated considering drought tolerance on the basis of PH (PH) in $\mathrm{cm}$, Leaf area (LA) in $\mathrm{cm}^{2}$, Leaf angle (La) in degree, chlorophyll contents (CC), leaf temperature (LT) in ${ }^{\circ} \mathrm{C}$, Days to silking (DS), Days to tasseling (DT), Anthesis to silking interval (ASI) in days, Cob length (CL) cm, Cob diameter (CD) in cm, Kernels rows per cob (KR),100 grains weight (GRW) in grams, Grain yield per plant (GYPP: grams). The data were recorded for 10 plants from each of the three replications.

\section{Biometrical and statistical analysis}

The general combining ability and specific combining ability was calculated according to Kempthorne (1957). Heterosis was calculated as Meredith and Bridge (1972) suggested.

\section{Molecular analysis}

The samples of the leaves were taken from the parents and their hybrids after two weeks of germination in the evaluation field. The samples were stored at $-80{ }^{\circ} \mathrm{C}$ for molecular analysis. The DNA extraction was done by modified CTAB method from each sample as described by Doyle and Doyle (1990). Quantification was done with the Nano Drop 2000 (spectrophotometer). The 1\% agarose gel was used to check the quality of DNA containing (EDTA/Tris/borate) buffer. About $3 \mu \mathrm{L}$ of bromophenol blue was added to parental DNA samples. Ten polymorphic SSR primers were selected from already available database as shown in Table 1. Working solutions both (parent and hybrid) contain $30 \mathrm{ng} / \mu \mathrm{l} \mathrm{sample.} \mathrm{The}$ 
reaction mixture for SSR analysis was $10 \mathrm{X}$ PCR buffer with $\left(\mathrm{NH}_{4}\right)_{2} \mathrm{SO}_{4}, \mathrm{MgCl}_{2}$, concentrations of genomic DNA, SSR primers and polymerase enzyme (taq polymerase), dNTPS (dATP, dCTP, dGTP, dTTP) were optimized. The master mixture for 10 parental and 21 hybrids contained $2.5 \mu \mathrm{L}$ of 10X PCR buffer, $2.5 \mu \mathrm{L} \mathrm{MgCl}_{2}, 1.0 \mu \mathrm{L}$ dNTPs, $0.2 \mu \mathrm{L}$ of taq polymerase, $1.0 \mu \mathrm{L}$ of forward primer, $1.0 \mu \mathrm{L}$ of reverse primer in $14.8 \mu \mathrm{L}$ of d3 $\mathrm{H}_{2}$ O. $2 \mu \mathrm{L}$ of diluted DNA was mixed with the master mixture in PCR tube. Initial denaturation was carried out at $94{ }^{\circ} \mathrm{C}$ for $5 \mathrm{~min}$, subsequent cycle consisted of denaturation at $94{ }^{\circ} \mathrm{C}$ for $1 \mathrm{~min}$, primer annealing at $45-60{ }^{\circ} \mathrm{C}$ for $1 \mathrm{~min}$, primer extension at $71{ }^{\circ} \mathrm{C}$ for 2 min, final extension at $71^{\circ} \mathrm{C}$ for $7 \mathrm{~min}$. When all the samples were uploaded, gel was run at $100 \mathrm{~V}$ for $2 \mathrm{~h}$. The gel was examined under gel documentation system GDC and photographed. Polymorphism was determined through visualization of the presence and absence of bands.

Table 1. Hybrids used for evaluation under water deficit condition

\begin{tabular}{|c|c|c|c|c|c|}
\hline & Testers & & Lines & & \\
\hline 1 & Golden & 1 & A50 & & \\
\hline 2 & Agati85 & 2 & A545 & & \\
\hline \multirow[t]{6}{*}{3} & EV189 & 3 & AE204 & & \\
\hline & & 4 & OH33-1 & & \\
\hline & & 5 & WM13RA & & \\
\hline & & 6 & ML13 & & \\
\hline & & 7 & Ml17 & & \\
\hline & Hybrids & & Hybrids & & Hybrids \\
\hline 1 & A50- $2 \times$ Golden & 8 & AES204×Agati85 & 15 & WM13RA $\times$ Ev189 \\
\hline 2 & A50-2×Agati85 & 9 & AES204×Ev189 & 16 & ML3×Golden \\
\hline 3 & A50-2×Ev189 & 10 & OH33-1×Golden & 17 & ML3×agati85 \\
\hline 4 & A545×Golden & 11 & OH33-1×Agati85 & 18 & ML3×Ev189 \\
\hline 5 & A545×Agati85 & 12 & OH33-1×Ev189 & 19 & ML17×Golden \\
\hline 6 & A545×Ev189 & 13 & WM13RA ×Golden & 20 & ML17×Agati85 \\
\hline 7 & AES204×Golden & 14 & WM13RA $\times$ Agati85 & 21 & ML17×Ev 189 \\
\hline
\end{tabular}

\section{Results and discussion}

\section{Genetic variability}

The analysis variance for the line $\times$ tester mating design for each trait was conducted under water deficit condition and their mean of square were given in Table 2. The genotypes showed highly significant contrast for PH, LA, La, CC, LT, KR, CL, CD, GRW, GYPP and significant in DS days to tasseling, and ASI. The parent showed prominent differences for most of the attribute except LA, DS, and DT. The crosses showed significant difference for all the traits except DT. The interaction parents vs crosses showed significant difference for $\mathrm{PH}, \mathrm{CD}$ and $\mathrm{KR}$. The lines revealed significant difference for most of the traits except La, LT, DS, ASI, GYPP. Tester showed significant difference for La, CC, ASI, $\mathrm{CD}, \mathrm{KR}, \mathrm{GYPP}$. The interaction line $\times$ tester showed significant difference for most of the traits PH, LA, La, LT, DS, ASI, CL, CD GYPP. It revealed that variability was present among the genotypes for various traits (Table 3). 
Table 2. List of simple sequence repeats (SSR) primers

\begin{tabular}{c|c|c|c}
\hline & Name & Forward primer $\left(\mathbf{5}^{\prime}\right.$-3') & Reverse (5'-3') \\
\hline 1 & bnlg439w1 & AGTTGACATCGCCATCTTGGTGAC & GAACAAGCCCTTAGCGGGTTGTC \\
2 & umc2007y4 & TTACACAACGCAACACGAGGC & GCTATAGGCCGTAGCTTGGTAGACAC \\
3 & bnlg1940k7 a & CGTTTAAGAACGGTTGATTGCATTCC & GCCTTTATTTCTCCCTTGCTTGCC \\
4 & UMC1363 & AAAGGCATTATGCTCACGTTGATT & TCTCCCTCCCCTGTACATGAATTA \\
5 & UMC1004 & CTGGGCATACAAAGCTCACA & TGCATAAACCGTTTCCACAA \\
6 & UMC2002 & TGACCTCAACTCAGAATGCTGTTG & CACAAAATCCTCGAGTTCTTGATTG \\
7 & Phi053 & CTGCCTCTCAGATTCAGAGATTGAC & AACCCAACGTACTCCGG \\
8 & Umc1600 & CGATCAGTGCGTGGAGAGTA & TAGGCATGCATTGTCCATTG \\
9 & umc1166 & CGATCAGATCATACACAACCTTGC & GAGGATCGATTCTTGGCGAGT \\
10 & umc1859k1 & AATCTCCAGGTTGGTGTTCAAAGG & AAAGATGACTTTGTGGGCAGTGG \\
\hline
\end{tabular}

\section{Combining ability analysis}

Identifying the highest performing lines and lines that can be used as parents in future crossings are two objectives in most crop breeding programs. Assessment of GCA and SCA effects is necessary to identify and select the better performing inbred lines and F1 crosses for each trait improvement (Areous et al., 2005; Danish et al. 2020, Ivy and Howlader, 2000; Oakey, 2006). General combining ability GCA can be defined as the performance of genotypes in the series of crosses while the SCA as the production in specific cross (Sprague and Tatum, 1942). The line WM13RA had high GC effect among the lines for La, CL, KR, GRW, GYPP and poor general combiner for LA, DT and DS. The line OH33-1 showed high GC effect for LA, CC, DT and low GC effect for ASI. The lineML17 had high GC effect for the LT while general combiner for La. The line ML3 had high GC effect for PH, DS, ASI and KR. The line A545 showed high GC effect among lines for CD. The line AES204 was noted low GC effect for PH. The line A50-2 proved to be poor combiner among the lines for CC, LT, CL, CD, GRW, GYPP (Tables 3 and 4). Specific combining ability WM13RA $\times$ EV189 top ranked among the negative SCA effects best for the reduction of PH. A545 $\times$ EV189 showed maximum negative SCA and proved to be good specific combiners for the leaf area. The cross ML $17 \times$ Ev189 displayed the highest negative and significant SCA declared as good specific combiner for La. The cross ML3 $\times$ EV189 was found the highest positive and significant SCA was declared as good specific combiner for CC. The cross A50-2 $\times$ Ev189 Table 5 was found to have maximum negative and significant SCA for the LT and revealed to be good specific combiners. The Cross combination ML3 $\times$ Agati85 displayed the highest negative and significant SCA, parent revealed to be good specific combiners for DS. The cross AES204 $\times$ Agati85 displayed the highest negative and significant SCA, and proved to be good specific combiner for DT. The Cross combination ML3 $\times$ Agati85 showed the maximum negative and significant SCA effects, and proved to be good specific combiner for ASI. The cross AES204 $\times$ Golden top ranked among positive and non -significant SCA, and revealed as good specific combiner for CL. The cross A545 $\times$ Ev189 showed the highest positive and significant SCA, and for $\mathrm{CD}$ it was a good specific combiner. The cross WM13RA $\times$ Golden showed the maximum positive and significant SCA for KR. The crossOH33-1 $\times$ Ev189 was found to have the highest positive and non-significant SCA and revealed as best specific combiners for GRW. The crossML17 $\times$ Agati85 showed the maximum positive and significant SCA for GYPP (Table 5) The results were in line with the results of Asif et al. (2020), Bibi et al. (2018), Kumar et al. (2004, 2016), Muraya et al. (2006), Uddin et al. (2008), Amiruzzaman et al. (2013), Gissa et al. (2013), Aminu et al. (2015), El-Shamarkaet al. (2015). 
Table 3. Mean square values of line $X$ tester of various traits under water deficit conditions

\begin{tabular}{|c|c|c|c|c|c|c|c|c|c|c|c|c|c|c|}
\hline Crosses & DF & $\begin{array}{l}\text { Plant height } \\
\text { (cm) }\end{array}$ & $\begin{array}{c}\text { Leaf area } \\
\left(\mathrm{cm}^{2}\right)\end{array}$ & $\begin{array}{l}\text { Leaf } \\
\text { angle }\end{array}$ & $\begin{array}{c}\text { Chlorophyll } \\
\text { contents } \\
\left(\mathrm{mgg}^{-1} \text { fr.wt). }\right.\end{array}$ & \begin{tabular}{|c|}
$\begin{array}{c}\text { Leaf } \\
\text { temperature } \\
\left({ }^{\circ} \mathbf{C}\right)\end{array}$ \\
\end{tabular} & $\begin{array}{c}\text { Days to } \\
\text { silking }\end{array}$ & $\begin{array}{c}\text { Days to } \\
\text { anthesis }\end{array}$ & $\begin{array}{c}\text { Anthesis to } \\
\text { silking } \\
\text { interval } \\
\end{array}$ & $\begin{array}{c}\text { Cob } \\
\text { length } \\
(\mathrm{cm})\end{array}$ & $\begin{array}{c}\begin{array}{c}\text { Cob } \\
\text { diameter } \\
(\mathbf{c m})\end{array} \\
\end{array}$ & $\begin{array}{l}\text { Kernels } \\
\text { per row }\end{array}$ & $\begin{array}{l}100 \text { grain } \\
\text { weight (g) }\end{array}$ & $\begin{array}{c}\text { Grain yield } \\
\text { per plant }(g)\end{array}$ \\
\hline Replication & 2 & $469.89 \mathrm{NS}$ & $726.8 \mathrm{NS}$ & $12.20 \mathrm{NS}$ & $11.25 \mathrm{NS}$ & $1.01 \mathrm{NS}$ & $1.31 \mathrm{NS}$ & $0.28 \mathrm{NS}$ & $0.29 \mathrm{NS}$ & $1.59 \mathrm{NS}$ & $0.1 \mathrm{NS}$ & $0.75 \mathrm{Ns}$ & $6.5 \mathrm{NS}$ & \\
\hline Genotype & 30 & $1452.9 * *$ & $11525 * *$ & $50.66^{* *}$ & $38.70 * *$ & $10.38 * *$ & $1.32 *$ & $1.10^{*}$ & $0.5959 *$ & $31.61 * *$ & $0.7 * *$ & $2.62 * *$ & $22 * *$ & $12341.6^{* *}$ \\
\hline Parents & 9 & $1708 * *$ & $4587 \mathrm{NS}$ & $62.01 * *$ & $51.82 * *$ & $8.57 * *$ & $0.99 \mathrm{NS}$ & $1.03 \mathrm{NS}$ & $0.732 *$ & $33.12 * *$ & $1 * *$ & $3.87 * *$ & $34 * *$ & $7792.7 * *$ \\
\hline Parent vs crosses & 1 & $8462 * *$ & $3413 \mathrm{NS}$ & $14.02 \mathrm{NS}$ & $0.19 \mathrm{NS}$ & $1.54 \mathrm{NS}$ & $1.90 \mathrm{NS}$ & $0.01 \mathrm{NS}$ & $0.72 \mathrm{NS}$ & $14.59 \mathrm{NS}$ & $0.70 *$ & $4.03 *$ & $5.7 \mathrm{NS}$ & $246.3 \mathrm{NS}$ \\
\hline Crosses & 20 & $987.38 * *$ & $15052 * *$ & $47.33^{* *}$ & $34.73 * *$ & $11.64 * *$ & $1.44^{*}$ & $1.18^{*}$ & $0.53 \mathrm{NS}$ & $31.77 * *$ & $0.6^{* *}$ & $1.98 * *$ & $17 * *$ & $14993.3 * *$ \\
\hline Lines & 6 & $2181 . * *$ & $5963.6^{*}$ & $39.16 \mathrm{NS}$ & $41.33 *$ & $2.16 \mathrm{NS}$ & $0.59 \mathrm{NS}$ & $1.03 \mathrm{NS}$ & $0.82 *$ & $40.27 * *$ & $1.3 * *$ & $4.49 * *$ & $46^{* *}$ & 2877.2NS \\
\hline Tester & 2 & $30.60 \mathrm{NS}$ & 1532NS & $96.39 * *$ & $77.91 * *$ & $4.45 \mathrm{NS}$ & $2.33 *$ & $1.33 \mathrm{NS}$ & $0.77 \mathrm{NS}$ & $26.86 \mathrm{NS}$ & $0.7 * *$ & $3.81 * *$ & 9NS & $18102.8 * *$ \\
\hline LinesX tester & 12 & $550.01 *$ & $21850 * *$ & $43.33 * *$ & $24.23 \mathrm{NS}$ & $17.57 * *$ & $1.72 *$ & $1.23^{*}$ & $0.34 \mathrm{NS}$ & $28.35^{* *}$ & $0.32 *$ & $0.42 \mathrm{Ns}$ & $4.3 \mathrm{NS}$ & $20533.1 * *$ \\
\hline Error & 60 & 308.31 & 2312.9 & 19.24 & 14.14 & 1.72 & 0.78 & 0.64 & 0.33 & 11.65 & 0.16 & 0.91 & 7.48 & 2171.1 \\
\hline
\end{tabular}

Table 4. General combining ability of various traits of maize under water deficit condition

\begin{tabular}{|c|c|c|c|c|c|c|c|c|c|c|c|c|c|}
\hline Nes & $\begin{array}{c}\text { Plant } \\
\text { height }(\mathrm{cm})\end{array}$ & $\begin{array}{c}\text { Leaf area } \\
\left(\mathrm{cm}^{2}\right)\end{array}$ & $\begin{array}{l}\text { Leaf } \\
\text { angle }\end{array}$ & $\begin{array}{c}\text { Chlorophyll } \\
\text { contents } \\
\left(\mathrm{mgg}^{-1} \text { fr.wt }\right)\end{array}$ & $\begin{array}{c}\text { Leaf } \\
\text { temperature } \\
\left({ }^{\circ} \mathrm{C}\right) \\
\end{array}$ & $\begin{array}{c}\text { Days to } \\
\text { silking }\end{array}$ & $\begin{array}{c}\text { Days to } \\
\text { anthesis }\end{array}$ & $\begin{array}{l}\text { Anthesis to } \\
\text { silking interval }\end{array}$ & $\begin{array}{c}\text { Cob length } \\
(\mathrm{cm})\end{array}$ & $\begin{array}{l}\text { Cob diameter } \\
(\mathbf{c m})\end{array}$ & $\begin{array}{c}\text { Kernels } \\
\text { rows per cob }\end{array}$ & $\begin{array}{l}100 \text { grain } \\
\text { weight (g) }\end{array}$ & $\begin{array}{l}\text { Grain yield } \\
\text { per plant }(\mathrm{g})\end{array}$ \\
\hline A50-2 & $-16.3 *$ & -24.53 & \begin{tabular}{|l|}
-0.79 \\
\end{tabular} & $-3.99 *$ & $3.76^{*}$ & -0.12 & -0.174 & 0.2 & $-2.3^{*}$ & $-0.358 *$ & -0.244 & $-2.4^{*}$ & $-40.04 *$ \\
\hline A545 & 5.2 & 16.09 & -1.16 & 0.12 & 0.2 & -0.12 & -0.174 & 0.23 & 0.03 & $0.52 *$ & -0.941 & $-1.84 *$ & 29.112 \\
\hline AES204 & $15.4^{*}$ & -2.46 & 0.68 & 0.49 & $-0.98^{*}$ & -0.12 & -0.396 & 0.222 & 2 & $0.39 *$ & $1.465^{*}$ & 1.576 & 15.08 \\
\hline OH33-1 & $13.7 *$ & $-47.15^{*}$ & 0.5 & 1.45 & $-1.26^{*}$ & -0.12 & -0.5 & 0.33 & -1.58 & 0.16 & 0.021 & 0.601 & -17.769 \\
\hline WM13RA & 7.85 & $54.33^{*}$ & -1.9 & 2 & -0.28 & $0.76^{*}$ & $0.82 *$ & -0.11 & $4.29 *$ & -0.22 & $2.391 *$ & $2.86^{*}$ & $58.48 *$ \\
\hline ML3 & $-26^{*}$ & 9.54 & 0.24 & 0.263 & -0.05 & -0.34 & 0.38 & $-0.44 *$ & -1.88 & -0.14 & -1.092 & -1.442 & -19.666 \\
\hline $\begin{array}{l}\text { ML17 } \\
\text { Tester }\end{array}$ & 0.076 & -5.82 & 2.42 & -0.33 & $-1.381 *$ & 0.095 & 0.047 & 0.24 & -0.51 & $0.34 *$ & -0.598 & 0.637 & -25.18 \\
\hline Agati85 & 0.85 & 9.22 & -0.27 & 1.28 & 0.435 & -0.031 & 0.015 & -0.09 & 0.042 & -0.05 & -0.488 & -0.718 & 14.45 \\
\hline Ev189 & 3.91 & -4.52 & -0.94 & -0.65 & -0.09 & -0.031 & 0.111 & -0.04 & 0.82 & -0.04 & 0.650 & -0.52 & -5.575 \\
\hline Golden & -4.76 & -4.7 & 1.21 & -0.63 & -0.34 & 0.063 & -0.12 & 0.14 & -0.86 & 0.104 & -0.162 & 0.19 & -8.879 \\
\hline
\end{tabular}


Table 5. Specific combining ability of various traits of maize under water deficit condition

\begin{tabular}{|c|c|c|c|c|c|c|c|c|c|c|c|c|c|}
\hline Hybrids & $\begin{array}{c}\text { Plant } \\
\text { height } \\
(\mathrm{cm})\end{array}$ & $\begin{array}{l}\text { Leaf } \\
\text { area } \\
\left(\mathrm{cm}^{2}\right)\end{array}$ & $\begin{array}{c}\text { Leaf } \\
\text { angle }\end{array}$ & $\begin{array}{c}\text { Chlorophyll } \\
\text { contents } \\
\left(\mathrm{mgg}^{-1} \text { fr.wt }\right)\end{array}$ & $\begin{array}{c}\text { Leaf } \\
\text { temperature } \\
\left({ }^{\circ} \mathrm{C}\right)\end{array}$ & $\begin{array}{c}\text { Days to } \\
\text { silking }\end{array}$ & $\begin{array}{c}\text { Days to } \\
\text { anthesis }\end{array}$ & $\begin{array}{c}\text { Anthesis } \\
\text { to silking } \\
\text { interval }\end{array}$ & $\begin{array}{c}\text { Cob } \\
\text { length } \\
(\mathrm{cm})\end{array}$ & $\begin{array}{c}\text { Cob } \\
\text { diameter } \\
(\mathbf{c m})\end{array}$ & $\begin{array}{c}\text { Kernels } \\
\text { rows per } \\
\text { cob }\end{array}$ & $\begin{array}{l}100 \text { grain } \\
\text { weight }(g)\end{array}$ & $\begin{array}{c}\text { Grain yield } \\
\text { per plant }(g)\end{array}$ \\
\hline A50-2× Golden & 2.04 & $58.57 *$ & $6.12 *$ & -0.5 & 1.16 & -0.301 & -0.015 & -0.23 & -1.74 & 0.398 & -0.28 & -1.3 & $74.213^{*}$ \\
\hline A50-2×Agati 85 & -5.51 & $57.57^{*}$ & $6.09 *$ & 2.74 & $2.53 *$ & 0.031 & -0.111 & 0.04 & -0.97 & -0.289 & -0.089 & 1.358 & 0.436 \\
\hline A50-2×Ev189 & 3.53 & -1.008 & -0.03 & -2.24 & -1.36 & 0.26 & 0.126 & 0.19 & 2.69 & -0.1 & 0.370 & -0.056 & $-74.64 *$ \\
\hline A545 $\times$ Golden & 5.27 & $139.13 *$ & -1.94 & -1.26 & -0.4 & 0.031 & -0.01 & 0.095 & 3.63 & -0.397 & 1.41 & 1.328 & $91.23 *$ \\
\hline A545×Agati 85 & -14.15 & -49.73 & -1.28 & 0.58 & 0.88 & 0.36 & 0.222 & 0.047 & -2.88 & -0.398 & -0.169 & -0.667 & -31.433 \\
\hline A545×Ev189 & 8.88 & -89.39 & $3.22 *$ & 0.68 & -0.48 & -0.39 & -0.2 & -0.14 & -0.74 & $0.795^{*}$ & -1.246 & -0.661 & $-59.8^{*}$ \\
\hline AES204×Golden & -9.79 & -16.9 & -2.42 & 0.54 & -0.38 & -0.301 & -0.12 & -0.12 & -3.46 & -0.063 & $-3.88^{*}$ & -0.564 & -20.749 \\
\hline AES204×Agati85 & 6.62 & -71.09 & -1.02 & -3.71 & -0.26 & 0.365 & -0.22 & 0.49 & 3.51 & 0.385 & 2.98 & 0.052 & -48.631 \\
\hline AES204×Ev189 & 3.16 & $87.99 *$ & -1.4 & 3.16 & 0.65 & -0.06 & 0.34 & -0.36 & -0.052 & -0.321 & 0.9 & 0.511 & $69.38 *$ \\
\hline OH33-1×Golden & -12.61 & $62.78^{*}$ & -1.94 & -2.22 & -0.26 & 0.031 & -0.015 & 0.095 & 2.94 & -0.212 & $2.22 *$ & -1.74 & -53.086 \\
\hline OH33-1×Agati85 & 1.54 & -14.28 & 3.71 & 0.15 & 0.64 & 0.031 & -0.11 & 0.04 & -3.6 & 0.253 & -1.57 & -0.876 & 12.619 \\
\hline OH33-1×Ev189 & 11.07 & 48.5 & -1.77 & 2.07 & -0.37 & -0.063 & 0.126 & -0.142 & 0.66 & -0.04 & -0.652 & 2.616 & 40.467 \\
\hline WM13RA $\times$ Golden & 6.29 & -30.4 & $-5.4^{*}$ & 2.25 & -0.81 & -0.52 & -0.34 & -0.12 & -1.55 & 0.181 & $2.96^{*}$ & 1.185 & -27.466 \\
\hline WM13RA $\times$ Agati85 & 11.13 & $83.67^{*}$ & -0.53 & 2.09 & -0.21 & $1.14^{*}$ & $1.22 *$ & -0.174 & 2.97 & -0.315 & -0.612 & 1.05 & $61.279 *$ \\
\hline WM13RA×Ev189 & -17.43 & -53.27 & $5.96^{*}$ & $-4.35^{*}$ & 1.02 & -0.61 & -0.87 & 0.3 & -1.41 & 0.134 & -2.35 & -2.241 & -33.813 \\
\hline ML3 $\times$ Golden & 18.14 & -7.372 & -2.02 & -1.42 & -0.31 & 0.92 & 0.42 & 0.206 & 0.15 & -0.031 & -1.21 & -1.305 & 33.143 \\
\hline ML3×agati85 & -4.65 & -5.16 & 1.2 & -3.61 & 1.24 & -1.74 & -0.33 & $-0.84^{*}$ & -0.62 & 0.024 & -1.35 & -0.821 & $-94.99 *$ \\
\hline ML3×Ev189 & -13.48 & 12.54 & 0.81 & $5.04 *$ & -0.93 & 0.82 & -0.095 & 0.63 & 0.47 & 0.007 & 2.57 & 2.127 & $61.85^{*}$ \\
\hline ML17×Golden & -9.34 & $-80.25^{*}$ & 2.79 & 2.61 & 1.02 & 0.14 & 0.095 & 0.095 & -0.001 & 0.125 & -1.23 & 2.397 & $-97.29 *$ \\
\hline ML17×Agati85 & 5.08 & $85.6^{*}$ & 4.01 & 1.75 & 0.23 & -0.19 & -0.66 & 0.38 & 1.61 & 0.34 & 0.82 & -0.101 & $100.72 *$ \\
\hline ML17×Ev 189 & 4.26 & -5.35 & $-6.8^{*}$ & $-4.37 *$ & -1.25 & 0.04 & 0.57 & -0.47 & -1.612 & -0.465 & 0.410 & -2.296 & -3.429 \\
\hline
\end{tabular}




\section{Heterosis and better parent heterosis}

The cross combination ML3 $\times$ Ev189 maximum mid parent negative heterosis and the cross combination ML3 $\times$ Ev189 showed the maximum negative heterobeltiosis for PH. The cross combination ML17 $\times$ Golden maximal negative mid parent heterosis estimate and the cross ML17 $\times$ Golden showed the maximal positive better parent heterotic effects for leaf area. The cross combination WM13RA $\times$ Golden showed minimal mid parent negative heterosis and Cross WM13RA $\times$ Golden showed minimal better parent heterotic effects for La (Ali et al., 2014; Malook et al., 2016; Mahmood et al., 2019; Paul and Duara, 1991; Yaqoob et al. 2020). The cross combination 5 ML3 $\times$ Ev189 maximal positive mid parent heterosis estimate and, the cross WM13RA $\times$ Golden showed the maximal positive better parent heterosis for CC. ML17 $\times$ Ev189 showed maximum negative mid parent heterosis and the cross combination ML17 $\times$ Ev189 showed maximum negative better parent heterosis for LT (Aslam et al., 2012). ML3 $\times$ Agati85 showed maximum negative mid parent heterosis and Cross ML3 $\times$ Agati85 showed maximum better parent heterosis for DS. In developing early mature, high yield hybrids early silking is considered as basis of breeding. So early flowering can be useful to escape from water scarcity at critical stage of crop development. AES204 $\times$ Golden showed maximum negative mid parent heterosis and the cross combination Cross OH33-1 × Golden and AES204 $\times$ Golden both showed maximum better parent heterosis for DT. ML3 $\times$ Agati85 showed maximum negative mid parent heterosis and Cross ML3 $\times$ Agati85 showed the highest better parent heterosis for ASI. The cross combination WM13RA $\times$ Agati 85 maximal positive mid parent heterosis estimate and the cross WM13RA $\times$ Agati 85 showed maximal positive better parent heterotic effects for CL. The cross combination A545 $\times$ Ev189 maximal positive mid parent heterosis estimate and the cross A545 $\times$ Ev189 showed maximal positive better parent heterotic effects for $\mathrm{CD}$. The cross combination $\mathrm{OH} 33-1 \times$ Golden showed maximal positive mid parent heterosis estimate. The cross combination OH33 -1 × Golden showed maximal positive better parent heterotic effects for KR. The cross combination AES204 $\times$ Ev189 had maximal positive mid parent heterosis estimate for GRW and GYPP. The cross AES204 × Ev189 showed maximal positive heterobeltiosis for GRW. Cross WM13RA $\times$ Agati showed maximal heterobeltiosis for GYPP (Tables 6 and 7).

\section{Molecular analysis}

The primer UMC 2002 was found polymorphic out of the ten SSRs primers used in the study. Hybrids confirmed on the presence and absence of the band in the gel for parents as well as for the hybrid. It was found that the hybrids of lines A50-2 $\times$ Golden showed the polymorphism and same fragment was found in the hybrid which confirm the inheritance of same fragment from the parent to offspring. The hybrid A50-2 $\times$ Golden was confirmed similarly for all the hybrid confirmed except for four hybrids AES $204 \times$ Golden, OH33-1 × Golden, WM13RA × Ev189 and ML3 × Golden. These hybrids can be confirmed by applying more number of primers and extensive screening of primer 6 is needed to identify the polymorphic fragment in both parents that can be finally detected in the hybrid population (Figure 1). The hybrid genetic purity was found $85.95 \%$ (Table 8) (Bibi et al., 2015; Hafeez et al., 2015; Farooq et al., 2017; Yaqoob et al. 2020). 
Table 6. Better parent heterosis of various traits under water deficit condition

\begin{tabular}{|c|c|c|c|c|c|c|c|c|c|c|c|c|c|}
\hline Hybrids & $\begin{array}{c}\text { Plant } \\
\text { height }(\mathrm{cm})\end{array}$ & $\begin{array}{c}\text { Leaf area } \\
\left(\mathrm{cm}^{2}\right)\end{array}$ & $\begin{array}{c}\text { Leaf } \\
\text { angle }\end{array}$ & $\begin{array}{c}\text { Chlorophyll } \\
\text { contents } \\
\left(\mathrm{mgg}^{-1} \text { fr.wt }\right)\end{array}$ & $\begin{array}{c}\text { Leaf } \\
\text { temperature } \\
\left({ }^{\circ} \mathrm{C}\right)\end{array}$ & $\begin{array}{l}\text { Days } \\
\text { to } \\
\text { silking }\end{array}$ & $\begin{array}{l}\text { Days to } \\
\text { anthesis }\end{array}$ & $\begin{array}{c}\text { Anthesis } \\
\text { to silking } \\
\text { interval }\end{array}$ & $\begin{array}{c}\text { Cob } \\
\text { length } \\
(\mathrm{cm})\end{array}$ & $\begin{array}{c}\text { Cob } \\
\text { diameter } \\
(\mathrm{cm})\end{array}$ & $\begin{array}{l}\text { Kernels } \\
\text { per row }\end{array}$ & $\begin{array}{l}100 \text { grain } \\
\text { weight (g) }\end{array}$ & $\begin{array}{l}\text { Grain } \\
\text { yield per } \\
\text { plant }(\mathrm{g})\end{array}$ \\
\hline A50-2× Golden & -3.16 & -1.85 & 9.01 & -1.49 & $25.11 *$ & $-2.65^{*}$ & -1.38 & -21.67 & $-16.87 *$ & -6.31 & 6.78 & $-23.1 *$ & -0.67 \\
\hline A50-2×Agati 85 & -8.73 & $-39.76^{*}$ & $-27.58 *$ & -4.81 & -2.24 & -0.45 & 0.15 & -22.22 & -8.07 & $-18.22 *$ & $-8.69 *$ & -5.24 & $-39.06^{*}$ \\
\hline A50-2×Ev189 & -4.99 & -23.30 & -2.05 & -4.23 & $21.39 *$ & 0.3 & -0.46 & 4.43 & -1.29 & 8.8 & -1.67 & -13.23 & $-71.15^{*}$ \\
\hline A $545 \times$ Golden & 12.30 & $56.84^{*}$ & -16.38 & 1.86 & -5.92 & $-2.21 *$ & -1.38 & 0.05 & 4.13 & -4.5 & 0.77 & -8.18 & $55.65^{*}$ \\
\hline A545×Agati 85 & -0.85 & -8.09 & -15.25 & -2.71 & -0.88 & -0.14 & -0.31 & -22.22 & -6.68 & -0.82 & $-10.15^{*}$ & -11.91 & -7.3 \\
\hline A545×Ev189 & 11.91 & $-22.45^{*}$ & 5.09 & 1.88 & $-11.6^{*}$ & -1.04 & -1.23 & 4.94 & -4.84 & $40.71 *$ & 3.9 & 0.65 & -23.08 \\
\hline AES204×Golden & 9.31 & -3 & -12.57 & -6.19 & $-16.53 *$ & $-2.65^{*}$ & $-1.84 *$ & 1.04 & -9.79 & -0.072 & -5.44 & -1.15 & -3.99 \\
\hline AES204×Agati85 & $17.91 *$ & $-18.70^{*}$ & $-23.15^{*}$ & $-12.4 *$ & $-14.82 *$ & $-0.44 *$ & -1.38 & 0.05 & $17.24 *$ & $10.68 *$ & -1.43 & $7.11 *$ & $-18.51 *$ \\
\hline AES204×Ev189 & $14.71 *$ & $41.38^{*}$ & $-18.58^{*}$ & $5.49 *$ & $-4.24 *$ & $-0.89 *$ & -0.92 & 0.05 & $15.32 *$ & -1.46 & $11.62 *$ & $25.04 *$ & $41.81^{*}$ \\
\hline OH33-1×Golden & 6.50 & $34.059 *$ & -11.36 & 0.98 & -4.24 & $-2.21^{*}$ & $1.84^{*}$ & 2.33 & -3.34 & -10.55 & $18.88^{*}$ & -11.65 & $-34.85^{*}$ \\
\hline OH33-×Agati85 & 13.82 & -1.2 & 21.56 & $-1.74 *$ & $-10.85^{*}$ & $0.89 *$ & -0.93 & $-11.11^{*}$ & $-18.79 *$ & $-0.49 *$ & $-10.1^{*}$ & $-2.01 *$ & $-1.64 *$ \\
\hline OH33-1×Ev189 & $18.67^{*}$ & 16.67 & 9.81 & 3.63 & -10.85 & -0.89 & -0.93 & 2.43 & $11.88 *$ & -3.47 & -0.071 & 12.51 & 36.19 \\
\hline WM13RA $\times$ Golden & $14.58 *$ & 11.84 & $-29.09 *$ & $5.95^{*}$ & $-16.14 *$ & $-1.76^{*}$ & -0.46 & -14.28 & 1.6 & -8.2 & 7.5 & 12.89 & 13.36 \\
\hline WM13RA $\times$ Agati85 & $16.035 *$ & $55.07^{*}$ & -10.7 & 0.93 & $-15.65^{*}$ & $2.25^{*}$ & $2.79 *$ & $-33.33^{*}$ & $22.22 *$ & -15.88 & -2.89 & $17.68^{*}$ & $55.82 *$ \\
\hline WM13RA $\times$ Ev19 & -2.93 & 5.22 & 17.14 & -3.47 & -9.4 & 0.05 & -0.46 & 14.28 & $18.29 *$ & 4.42 & $15.92 *$ & 10.76 & 6.07 \\
\hline ML3 $\times$ Golden & -11.82 & -0.67 & -12.38 & -0.45 & $-11.02 *$ & -1.32 & 0.05 & -14.28 & -10.51 & -10.96 & $-10.80^{*}$ & $-34.6^{*}$ & 0.62 \\
\hline ML3×agati85 & $-22.57 *$ & -4.44 & 4.78 & -7.07 & -4.54 & $-3.15^{*}$ & 0.93 & $-66.66 *$ & -5.71 & -7.77 & $-13.52 *$ & $-28.3^{*}$ & $-66.54 *$ \\
\hline ML3×Ev189 & $-32.10^{*}$ & 1.27 & 10.66 & 4.54 & $-19.88^{*}$ & 0.9 & 0.01 & 26.38 & -5.1 & -4.74 & $-14.19^{*}$ & $-18.6^{*}$ & 3.06 \\
\hline ML17×Golden & -0.46 & $-33.88^{*}$ & 8.68 & 1.85 & -4.38 & $-1.76^{*}$ & -0.92 & $-36.36^{*}$ & -8.41 & $-11.9^{*}$ & 5.08 & $-15.5^{*}$ & $-63.5^{*}$ \\
\hline ML17×Agati85 & 7.67 & 12.6 & $29.4^{*}$ & -1.93 & $-13.34 *$ & -0.89 & 0.04 & $-27.27 *$ & -1.99 & -3.61 & -4.34 & $-20.0 *$ & 12.02 \\
\hline ML17×Ev 189 & 4.48 & -15.25 & -1.17 & -7.8 & $-25.22 *$ & -0.44 & 0.465 & $-45.45^{*}$ & -15.17 & -8.44 & -1.67 & $-29.1 *$ & $-33.57 *$ \\
\hline
\end{tabular}

APPLIED ECOLOGY AND ENVIRONMENTAL RESEARCH 18(2):3511-3523.

http://www.aloki.hu • ISSN 15891623 (Print) • ISSN 17850037 (Online)

DOI: http://dx.doi.org/10.15666/aeer/1802_35113523

๑ 2020, ALÖKI Kft., Budapest, Hungary 
Table 7. Heterosis of various various traits under water deficit conditions

\begin{tabular}{|c|c|c|c|c|c|c|c|c|c|c|c|c|c|}
\hline Hybrids & $\begin{array}{l}\text { Plant } \\
\text { height } \\
(\mathbf{c m})\end{array}$ & $\begin{array}{l}\text { Leaf } \\
\text { area } \\
\left(\mathrm{cm}^{2}\right)\end{array}$ & $\begin{array}{c}\text { Leaf } \\
\text { angle }\end{array}$ & $\begin{array}{c}\text { Chlorophyll } \\
\text { contents } \\
\left(\mathrm{mgg}^{-1} \text { fr.wt }\right) .\end{array}$ & $\begin{array}{c}\text { Leaf } \\
\text { temperature } \\
\left({ }^{\circ} \mathrm{C}\right)\end{array}$ & $\begin{array}{c}\text { Days to } \\
\text { silking }\end{array}$ & $\begin{array}{l}\text { Days to } \\
\text { anthesis }\end{array}$ & $\begin{array}{c}\text { Anthesis } \\
\text { to silking } \\
\text { interval }\end{array}$ & $\begin{array}{l}\text { Cob } \\
\text { length } \\
(\mathrm{cm})\end{array}$ & $\begin{array}{c}\text { Cob } \\
\text { diameter } \\
(\mathrm{cm})\end{array}$ & $\begin{array}{l}\text { Kernels } \\
\text { per row }\end{array}$ & $\begin{array}{c}100 \text { grain } \\
\text { weight }(\mathrm{g})\end{array}$ & $\begin{array}{c}\text { Grain yield } \\
\text { per plant } \\
\text { (g) }\end{array}$ \\
\hline A50-2× Golden & 7.62 & 6.035 & 10.69 & -0.76 & $35.99 *$ & $-1.63^{*}$ & -0.61 & -18.14 & $-14.22 *$ & $17.59 *$ & 6.78 & $-23.04 *$ & 6.53 \\
\hline A50-2×Agati85 & 2.75 & $-31.37 *$ & $-20.15^{*}$ & -1.77 & 7.13 & -0.3 & 0.31 & -15.96 & -7.54 & 1.25 & -1.55 & -5.21 & $-30.87 *$ \\
\hline A50-2×Ev189 & 5.21 & -11.05 & 16.4 & -1.74 & $43.12^{*}$ & 0.37 & -0.15 & 17.13 & 4.1 & $24.38 *$ & 1.19 & -6.7 & $-54.58 *$ \\
\hline A $545 \times$ Golden & $22.529 *$ & $60.79 *$ & $-15.81 *$ & 2.077 & 1.16 & $-1.41 *$ & -1.07 & 2.41 & 7.16 & 1.15 & 3.285 & 1.332 & $59.6^{*}$ \\
\hline A545 $\times$ Agati85 & 9.62 & -4.94 & 5.84 & -0.53 & 7.47 & -0.07 & 0.31 & -10.65 & -6.4 & 3.25 & -5.35 & -2.66 & -3.85 \\
\hline A $545 \times$ Ev 189 & $21.678 *$ & -18.17 & $25.77 *$ & $5.5^{*}$ & 3.21 & -0.75 & -1.08 & 10.49 & 0.61 & $48.81 *$ & 4.35 & 3.7 & 15.77 \\
\hline AES204×Golden & $34.776^{*}$ & 1.74 & -5.7 & -1.02 & -8.87 & $-2.004 *$ & $-1.61 *$ & 0.05 & 2.15 & 0.2915 & -1.22 & 6.05 & 1.061 \\
\hline AES204×Agati85 & $47.022 *$ & -17.85 & -8.2 & $-9.78^{*}$ & $-11.01 *$ & -0.224 & -0.69 & 12.5 & $29.75^{*}$ & $12.28 *$ & 1.88 & $15.07 *$ & -17.71 \\
\hline AES204×Ev189 & $41.03^{*}$ & $45.83^{*}$ & 4.0018 & 2.86 & 0.822 & -0.45 & -0.69 & 7.69 & $20.7 *$ & 9.64 & $13.34 *$ & $25.12 *$ & $110.53 *$ \\
\hline OH33-1×Golden & 7.92 & $-23.14^{*}$ & -4.32 & 1.84 & 2.58 & $-1.55^{*}$ & $-1.38 *$ & 6.66 & -2.75 & -9.42 & $20.86^{*}$ & -11.36 & -24.35 \\
\hline OH33-×Agati85 & $17.02 *$ & 9.2799 & $-26.26^{*}$ & -0.165 & 1.18 & 0.67 & -0.46 & 5.88 & $-16.17 *$ & 2.55 & -4.59 & -1.82 & 8.14 \\
\hline OH33-1×Ev189 & $19.79 *$ & 26.72 & $23.62 *$ & $7.965^{*}$ & -0.99 & -0.45 & -0.93 & 14.28 & -3.74 & 8.952 & 1.18 & $21.16^{*}$ & $90.145^{*}$ \\
\hline WM13RA $\times$ Golden & $23.60 *$ & 15.12 & $26.76^{*}$ & $7.165^{*}$ & -7.78 & -0.89 & 0.05 & -14.28 & $13.77 *$ & -3.51 & $8.40 *$ & 17.92 & 17.098 \\
\hline WM13RA $\times$ Agati85 & $26.86^{*}$ & $59.73^{*}$ & -3.09 & 2.25 & -6.49 & $2.25^{*}$ & $3.27 *$ & $-25^{*}$ & $33.7 *$ & $-13.1^{*}$ & 3.88 & $23.09 *$ & $60.454^{*}$ \\
\hline WM13RA×Ev189 & 4.32 & 10.6 & $37.23^{*}$ & 0.84 & 7.92 & 0.22 & -0.465 & 23.07 & $22.28 *$ & 11.27 & $18.35^{*}$ & 13.98 & $59.07 *$ \\
\hline ML3×Golden & -5.90 & 1.78 & -8.23 & 0.892 & -2.37 & 0.15 & 0.93 & -9.97 & -7.07 & $-10.08 *$ & -0.744 & $-27.49 *$ & 2.79 \\
\hline ML3×agati85 & $-18.50 *$ & 3.58 & 12.2 & $-6.05^{*}$ & 5.58 & $-2.56^{*}$ & 0.938 & $-60.86 *$ & -4.55 & -7.02 & $-10.50 *$ & $-20.51 *$ & $-63.71 *$ \\
\hline ML3×Ev189 & $-27.27 *$ & 11.9 & $28.08 *$ & $9.44 *$ & -4.75 & 1.28 & 0.467 & 29.76 & -0.54 & 5.4 & $-6.99 *$ & -3.76 & $58.64 *$ \\
\hline ML17×Golden & -0.23 & $-30.10^{*}$ & $17.32 *$ & 3.965 & 1.49 & -1.11 & 0.467 & $-22.22 *$ & -7.83 & -5.27 & 6.43 & -5.13 & $-61.51 *$ \\
\hline ML17×Agati85 & 9.01 & $25.68^{*}$ & $34.4^{*}$ & -1.561 & -7.22 & -0.67 & 0.47 & $-20 *$ & 1.19 & 1.91 & 4.35 & -10.34 & $25.106^{*}$ \\
\hline ML17×Ev 189 & 5.14 & -3.65 & 11.25 & -2.82 & $-13.7 *$ & 0.05 & $1.40 *$ & $-29.41 *$ & -7.31 & -4.66 & 2.46 & $-15.33 *$ & 3.78 \\
\hline
\end{tabular}


Table 8. Hybrid confirmation by UMC2002 SSR marker

\begin{tabular}{|c|c|c|c|c|}
\hline Sr no & Hybrid/parent & Parent 1 & Parent 2 & Confirmation \\
\hline 1 & A50- $2 \times$ Golden & + & + & Confirmed \\
\hline 2 & A50-2×Agati85 & + & + & Confirmed \\
\hline 3 & A50-2×Ev189 & + & + & Confirmed \\
\hline 4 & A545 $\times$ Golden & + & + & Confirmed \\
\hline 5 & A545 $\times$ Agati85 & + & + & Confirmed \\
\hline 6 & A545×Ev189 & + & + & Confirmed \\
\hline 7 & AES204×Golden & + & - & Not confirmed \\
\hline 8 & AES204×Agati85 & + & + & Confirmed \\
\hline 9 & AES204×Ev189 & + & + & Confirmed \\
\hline 10 & OH33-1×Golden & + & + & Not confirmed \\
\hline 11 & OH33-1×Agati85 & + & + & Confirmed \\
\hline 12 & OH33-1×Ev189 & + & + & Confirmed \\
\hline 13 & WM13RA×Golden & + & + & Confirmed \\
\hline 14 & WM13RA $\times$ Agati85 & + & + & Confirmed \\
\hline 15 & WM13RA×Ev189 & + & - & Not confirmed \\
\hline 16 & ML3×Golden & + & - & Not confirmed \\
\hline 17 & ML3×agati85 & + & + & Confirmed \\
\hline 18 & ML3×Ev189 & + & + & Confirmed \\
\hline 19 & ML17×Golden & + & + & Confirmed \\
\hline 20 & ML17×Agati85 & + & + & Confirmed \\
\hline 21 & ML17×Ev 189 & + & + & Confirmed \\
\hline \multicolumn{4}{|c|}{ Purity of hybrid } & 80.9524 \\
\hline
\end{tabular}

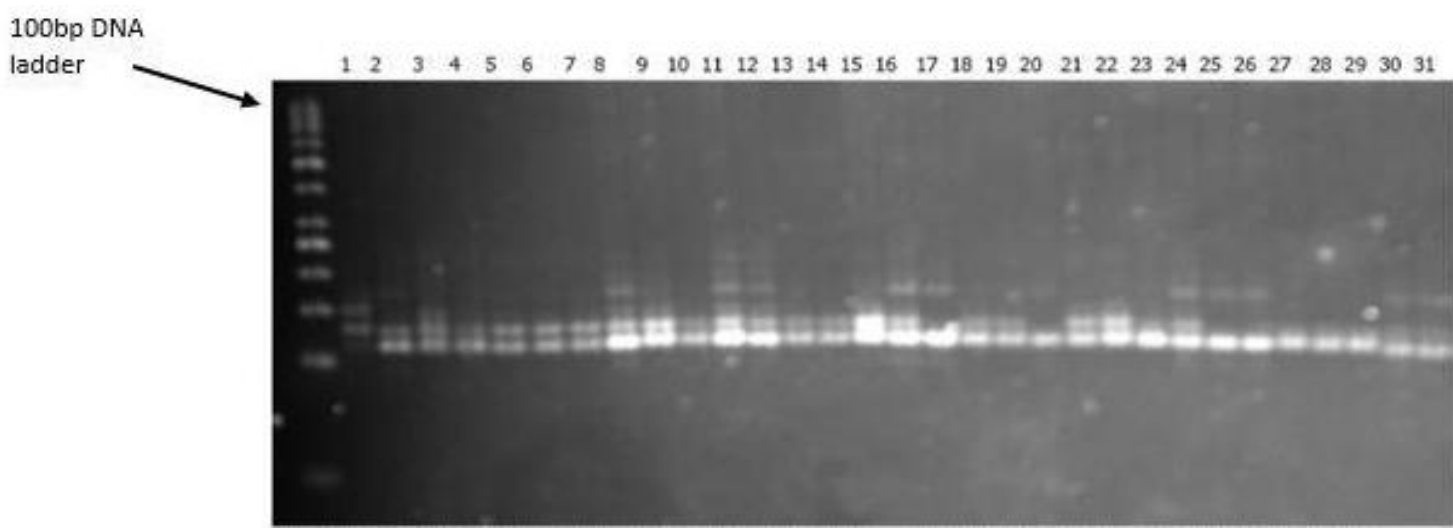

Figure 1. The lanes indicated lines, testers and hybrids; 1) Golden, 2) A50, 3) Agati85, 4) A545, 5) EV189, 6) AE204, 7) OH33-1, 8) WM13RA, 9) ML13, 10) Ml17, 11) A50-2 × Golden, 12) AES204 × Agati85, 13) WM13RA $\times$ Ev189, 14) A50-2 × Agati85, 15) AES204 × Ev189, 16) ML3 $\times$ Golden, 17) A50-2 × Ev189, 18) OH33-1 × Golden, 19) ML3 × Agati85, 20) A545 × Golden, 21) OH33-1 × Agati85, 22) ML3 × Ev189, 23) A545 × Agati85, 24) OH33-1 × Ev189, 25) ML17 × Golden, 26) A545 × Ev189, 27) WM13RA $\times$ Golden, 28) ML17 × Agati85, 29) AES204 × Golden, 30) WM13RA $\times$ Agati85, 31) ML17 $\times$ Ev 189 


\section{Conclusion}

It is a need among plant breeders to be able to select and develop tolerant genotypes for water deficit conditions. The research displayed that the line WM13RA and OH33-1 showed the highest and 6 general combining ability for most of the traits. The cross combination WM13RA $\times$ Agati85was found to have the highest positive better parents heterosis. Specific combing ability was also high in ML17 $\times$ Agati85 for grain yield and ML3 $\times$ Agati85 for the production of early maturing hybrids. The genetic purity of the hybrids was found about $80.95 \%$ in molecular analysis with SSR markers.

\section{REFERENCES}

[1] Aaliya, K., Qamar, Z., Nasir, I. A., Ali, Q., Munim, A. F. (2016): Transformation, evaluation of gtgene and multivariate genetic analysis for morpho-physiological and yield attributing traits in Zea mays. - Genetika 48(1): 423-443.

[2] Ali, F., Kanwal, N., Ahsan, M., Ali, Q., Bibi, I., Niazi, N. K. (2015): Multivariate analysis of grain yield and its attributing traits in different maize hybrids grown under heat and drought stress. - Scientifica. https://doi.org/10.1155/2015/563869.

[3] Ali, F., Ahsan, M., Ali, Q., Kanwal, N. (2017): Phenotypic stability of Zea mays grain yield and its attributing traits under drought stress. - Frontiers in Plant Science 8: 1397.

[4] Ali, Q., Ahsan, M., Ali, F., Aslam, M., Khan, N. H., Munzoor, M., Mustafa, H. S. B., Muhammad, S. (2013): Heritability, heterosis and heterobeltiosis studies for morphological traits of maize (Zea mays L.) seedlings. - Advancements in Life Sciences 1(1): 52-63.

[5] Ali, Q., Ali, A., Ahsan, M., Ali, S., Khan, N. H., Muhammad, S., Abbas, H. G., Nasir, I. A., Husnain, T. (2014): Line $\times$ tester analysis for morpho-physiological traits of Zea mays L. seedlings. - Advancements in Life Sciences 1(4): 242-253.

[6] Ali, Q., Ahsan, M., Malook, S., Kanwal, N., Ali, F., Ali, A., Ahmed, W., Ishfaq, M., Saleem, M. (2016): Screening for drought tolerance: comparison of maize hybrids under water deficit condition. - Advancements in Life Sciences 3(2): 51-58.

[7] Aminu, D., Garba, M., Muhammad, A. (2015): Combining ability and heterosis for phonologic and agronomic traits in maize (Zea mays L.) under drought conditions in the Northern Guinea Savanna of Borno State, Nigeria. - Afr. J. Biotechnol. 13(24): 2400-2406.

[8] Amiruzzaman, M., Islam, M. A., Hasan, L., Kadir, M., Rohman, M. (2013): Heterosis and combining ability in a diallel among elite inbred lines of maize. Zea mays L. - J. Food Agric. 25(2): 132-137.

[9] Anonymous (2019): Ministry of Food and Agriculture, Bureau of Statistics. Government of Pakistan, Islamabad.

[10] Areous, L., Slafer, G., Royo, C., Serret, M. D. (2005): Breeding for yield potential and stress adaptation in cereals. - Crit. Rev. Plant Sci. 27: 377-412.

[11] Asif S, Ali Q, Malik A. (2020): Evaluation of salt and heavy metal stress for seedling traits in wheat. -Biol. Clin. Sci. Res. J. 2020: e005.

[12] Aslam, M., Khan, J. A., Basra, S. M. A. (2012): Combing ability estimates ability estimates and mode of inheritance for drought related traits in genetically distant maize accessions (Zea mays L.). - Anim. Plant Sci. 22(3): 679-682.

[13] Bibi, T., Mustafa, H. S. B., Hasan, E. U., Rauf, S., Mahmood, T., Ali, Q. (2015): Analysis of genetic diversity in linseed using molecular markers. - Life Sci J 12(4): 28-37.

[14] Bibi, T., Mustafa, H. S. B., Mahmood, T., Hameed, A., Ali, Q. (2018): Multivariate analysis for adaptability and yield stability of rapeseed (Brassica napus L.) strains in different agro-climatic zones. - Genetika (0534-0012) 50(2): 369-378.

[15] Chaudhary, A. R. (1983): Maize in Pakistan. - Punjab Agri. Res. Coordination Board, Uni. Agri. Faisalabad, Pakistan. 
[16] Danish P, Ali Q, Hafeez MM, Malik A, (2020): Antifungal and antibacterial activity of aloe vera plant extract. -Biol. Clin. Sci. Res. J. 2020: e003.

[17] Dahmardeh, M., Ghanbari, A., Syasar, B., Ramroudi, M. (2009): Effect of intercropping maize with cowpea on green forage yield and quality evaluation. - Asian J. Plant Sci 8(3): 235-239.

[18] Doyle, J. J., Doyle, J. L. (1990): Isolation of plant DNA from fresh tissue. - Focus 12: 13-15.

[19] El-Shamarka, S. A., Ahmed, M. A., El-Nahas, M. M. (2015): Heterosis and combining ability for yield and its components through diallel cross analysis in maize, Zea mays L. Alex. J. Agric. Res. 60(2): 87-94.

[20] FAO (2019): Statistical Database. - http://faostat.fao.org.

[21] Farooq, A. M., Nasir, I. A., Ali, Q., Tabassum, B., Husnain T. (2017): Identification and interrelationship of yield related traits through DNA fingerprinting in Zea mays. International Journal of Biology, Pharmacy and Allied Sciences 6(6): 1276-1303.

[22] Gissa, D. W., Zelleke, H., Labuschagne, M. T., Hussien, T. Singh, H. (2013): Heterosis and combining ability for grain yield and its components in selected maize inbred lines. Afr. J. Plant Soil. 24(3): 133-137.

[23] Hafeez, M. N., Sadique, S., Hassan, S., Sarwar, M. B., Rashid, B., Ali, Q., Husnain, T. (2015): Physiological, morphological, biochemical and molecular basis of drought tolerance in cotton. - Int. J. Biol. Pharm. Allied Sci 4: 1091-1112.

[24] Ivy, N. A., Howlader, M. S. (2000): Combining ability in maize. - Bangladesh J. Agril. Res. 25(3): 385-92.

[25] Kepmthorne, O. (1957): An Introduction to Genetic Statistics. - John Wiley and Sons Inc., New York.

[26] Kumar, P., Gupta, S. (2004): Genetic analysis in maize (Zea mays L.). - J. Res. Birsa Agri. Univ. 16(1): 113-117.

[27] Kumar, S. V. V. P., Babu, D. R. (2016): Combining ability and heterosis in maize, Zea mays L. for grain yield and yield components. - IJAEB 9(5): 763-772.

[28] Mahmood, T., Mustafa, H. S. B., Aftab, M., Ali, Q., Malik, A. (2019): Super canola: newly developed high yielding, lodging and drought tolerant double zero cultivar of rapeseed (Brassica napus L.). - Genetics and Molecular Research 18(2): gmr16039951.

[29] Malook, S., Ali, Q., Ahsan, M., Shabaz, M., Waseem, M., Mumtaz, A. (2016): Combining ability analysis for evaluation of maize hybrids under drought stress. Journal of the National Science Foundation of Sri Lanka 44(2): 223-230.

[30] Meredith, W. E., Bridge, R. R. (1972): Heterosis and gene action in cotton Gossypium hirsutum L.). - Crop Sci. 12: 304-310.

[31] Mishra, V., Cherkauer, K. A. (2010): Retrospective droughts in the crop growing season: implications to corn and soybean yield in the mid-western united states. - Agr. Meteorol. 150: 1030-1045.

[32] Muraya, M. M., Ndirangu, C. M., Omolo, E. O. (2006): Heterosis and combining ability in diallel crosses involving maize (Zea mays L.) S1 lines. - Australian J. Exp. Agri. 46(4): 387-394.

[33] Oakey, H., Verbyla, A., Pitchford, W., Cullis, B., Kuchel, H. (2006): Joint modeling of additive and non-additive genetic line effects in single field trials. - Theor Appl Genet 113(5): 809-819.

[34] Paul, S. K., Duara, R. K. (1991): Combining ability studies in maize (Zea mays L.). Intl. adaptation in cereals. - Crit. Rev. Plant Sci. 27: 377-412.

[35] Rana, M. A. (2014): The seed industry in Pakistan: regulations, politics, and entrepreneurship. PSSP Working Paper 19, Washington, DC. - International Food Policy Research Institute (IFPRI) Res. 25: 385-392.

[36] Sprague, G. F., Tatum, L. A. (1942): General versus specific combining ability in single crosses of corn. - J. Americ. Soc. Agron. 34: 923-932. 
[37] Uddin, M. S., Amiruzzaman, M., Bagum, S. A., Hakim, M. A., Ali, M. R. (2008): Spring maize (Zea mays L.) S1 lines. - Aust J. Exp. Agri. 46(4): 387-394.

[38] Wynne, J. C., Emery, D. A., Rice, P. W. (1970): Combining ability estimates in Arachis hypogaea L. II. Field performance of F1 hybrids. - Crop Sci. 10: 713-715.

[39] Yaqoob S, Fatima N, Khan S, Ali Q, Hafeez MM, Malik A. (2020): Begomoviruses and betasatellites associated with CLCuD. - Biol. Clin. Sci. Res. J. 2020: e002. 